\title{
Rapid profiling of isomeric unsaturated lipids through online photochemical derivatization of $\mathbf{C}=\mathbf{C}$ bonds
}

Daisy Unsihuay ${ }^{[a]}$, Pei Su ${ }^{[a]}$, Jiamin Qiu ${ }^{[b]}$, Shihuan Kuang ${ }^{[b]}$, Yingju Li ${ }^{[c]}$, Xiaofei Sun ${ }^{[c]}$, Sudhansu K. Dey ${ }^{[\mathrm{c}]}$, and Julia Laskin ${ }^{[\mathrm{a}] *}$

[a] Department of Chemistry, Purdue University, West Lafayette, IN

[b] Department of Animal Sciences, Purdue University, West Lafayette, IN

[c] Division of Reproductive Sciences, Cincinnati Children's Hospital Medical Centre and Department of Pediatrics, University of Cincinnati College of Medicine, Cincinnati, OH

Abstract: Unraveling the complexity of the lipidome requires the development of novel approaches for the structural characterization of lipid species with isomeric resolution. Herein, we introduce an online photochemical approach for lipid isomer identification through selective derivatization of double bonds by reaction with singlet oxygen. Lipid hydroperoxide products are generated promptly after laser irradiation. Fragmentation of these species in a mass spectrometer produces diagnostic fragments, which reveal the $\mathrm{C}=\mathrm{C}$ locations in the unreacted lipids. This approach uses an inexpensive light source and photosensitizer making it easy to incorporate into any lipidomics workflow. We demonstrate the utility of this approach for the shotgun profiling of $\mathrm{C}=\mathrm{C}$ locations in different lipid classes present in tissue extracts using electrospray ionization (ESI) and for spatially-resolved analysis of lipids in tissue sections using nanospray desorption electrospray ionization (nano-DESI). These results provide a path for both rapid profiling and ambient imaging of positional isomers in biological samples.

Lipids are essential biomolecules acting as structural components of membranes, energy reservoirs, or signaling molecules in biological systems. ${ }^{[1]}$ Multiple biochemical transformations that lipids undergo during their biosynthesis generate diverse lipid structures, which complicates their structural characterization. ${ }^{[2]}$ Mass spectrometry (MS) has been extensively used to identify lipid classes, acyl chain composition, and degree of unsaturation ${ }^{[3]}$ However, the differentiation of isomers that vary only by the position of $\mathrm{C}=\mathrm{C}$ double bonds critical to understanding their role in key metabolic processes remains challenging. Recent studies have attributed changes in the relative abundance of positional isomers to altered lipid metabolism in cancer ${ }^{[4,5]}$ and diabetes. ${ }^{[6]}$ These findings inspire the development of new approaches for the identification of $\mathrm{C}=\mathrm{C}$ bond locations in unsaturated lipids. ${ }^{[7]}$

The most successful MS-based strategies for identifying $\mathrm{C}=\mathrm{C}$ bond positions include ozone-induced dissociation, ${ }^{[8,9]}$ ion-ion reactions, ${ }^{[10]}$ electron-ion reaction-based dissociation, ${ }^{[11,12]}$ and ultraviolet photodissociation. ${ }^{[13,14]}$ Alternatively, derivatization methods including Paternò Büchi, ${ }^{[15,16]}$ ozonolysis, ${ }^{[17]}$ epoxidation, ${ }^{[18,19]}$ and thiol-ene ${ }^{[20]}$ reactions have been used for the untargeted analysis of the double bond in unsaturated lipids thereby dramatically expanding the depth of molecular coverage obtained in lipidomics experiments. When coupled with mass spectrometry imaging (MSI), these approaches reveal the spatial localization of isomeric lipids in biological systems otherwise invisible with traditional MSI techniques. ${ }^{[5,21-24]}$ 
Despite the significant progress in this field, isomer-selected shotgun lipidomics and MSI experiments would substantially benefit from faster derivatization reactions and simplified sample preparation approaches. Coupling online derivatization with liquid extraction-based MSI is particularly advantageous for the untargeted quantitative analysis of biological samples without special sample pretreatment. Herein, we introduce an online derivatization approach, that 1) enables fast photooxidation and profiling of $\mathrm{C}=\mathrm{C}$ locations in lipids, 2) uses an inexpensive visible light source which can be easily focused, and 3) is compatible with both shotgun lipidomics workflows for the analysis of lipid extracts and direct analysis of biological samples using liquid extraction-based techniques. In particular, we focus on the development of isomer-specific profiling using electrospray ionization (ESI) and high-resolution nanospray desorption electrospray ionization (nano-DESI) MS.

Nano-DESI is an ambient ionization technique, in which analytes are directly extracted from a sample using a "V" shaped probe comprised of two capillaries and are subsequently ionized by ESI at a mass spectrometer inlet. ${ }^{[25]}$ The addition of appropriate reagents to the extraction solvent may be used to perform online chemical derivatization of analytes, ${ }^{[26]}$ which often benefits from reaction acceleration in microdroplets. ${ }^{[27]}$ Coupling of nano-DESI with the on-line photochemical reaction is a first step towards the development of a suite of derivatization approaches for isomer-selective imaging of lipids and metabolites in biological samples. The approach reported herein utilizes a reaction between singlet oxygen $\left({ }^{1} \mathrm{O}_{2}\right)$ with $\mathrm{C}=\mathrm{C}$ bonds and enables efficient derivatization of unsaturated lipids on a timescale of $\sim 100 \mathrm{~ms}$ making it compatible with imaging experiments.

${ }^{1} \mathrm{O}_{2}$ is the excited state of molecular oxygen containing one empty orbital, which reacts with electron-rich double bonds to produce hydroperoxides as shown in Figure 1a. ${ }^{[28,29]}$ Photochemical generation of ${ }^{1} \mathrm{O}_{2}$ is achieved using a photosensitizer, which is excited to a triplet state upon exposure to light and subsequently transfers its excitation energy to molecular oxygen present in solution. ${ }^{1} \mathrm{O}_{2}$ reacts with lipids to form lipid hydroperoxides (LOOHs). ${ }^{[30-32]}$ When subjected to collisioninduced dissociation (CID), LOOHs produce

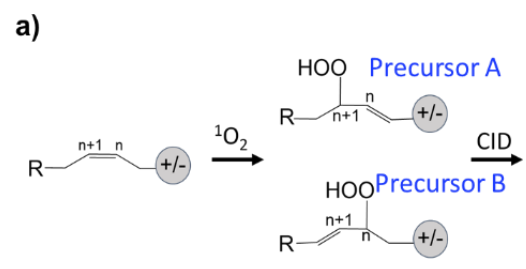

b)
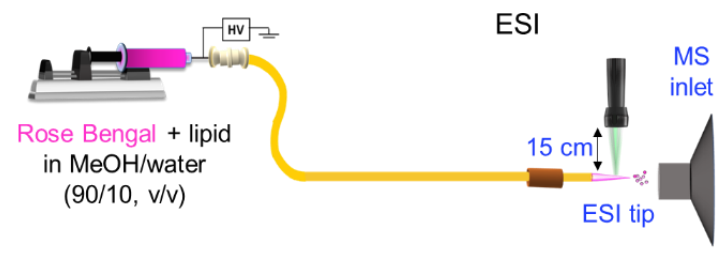

c)

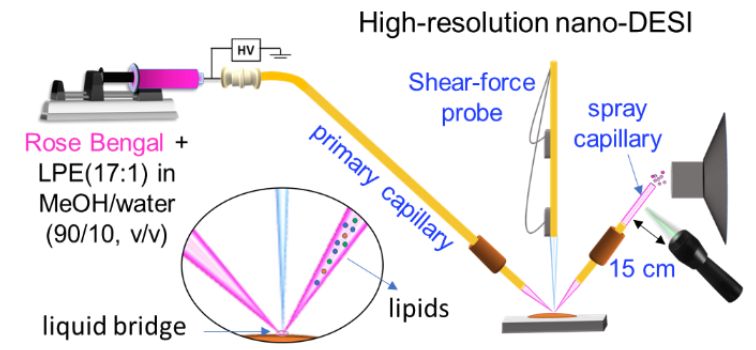

Figure 1. a) Schematic representation of the oxidation of the $\mathrm{C}=\mathrm{C}$ bonds in lipids by singlet oxygen and fragmentation via $\mathrm{CID}$ that yields unique neutral losses. Experimental setup for the online singlet oxygen reaction with lipids coupled to b) ESI and c) nano-DESI. 
fragments informative of the position of the hydroperoxide group. Herein, we use the on-line reaction of unsaturated lipids with ${ }^{1} \mathrm{O}_{2}$ followed by $\mathrm{CID}$ of the resulting $\mathrm{LOOH}$ products to obtain diagnostic peaks that provide insights into the $\mathrm{C}=\mathrm{C}$ bond location.

Proof-of-concept experiments were carried out using ESI as shown in Figure 1b and described in detail in the supporting information. In this experiment, a mixture of $\mathrm{MeOH}: \mathrm{H}_{2} \mathrm{O}$ (90/10, v/v) containing Rose Bengal (RB) as a photosensitizer and lipid analytes is propelled through a fused silica capillary at $0.5 \mu \mathrm{L} / \mathrm{min}$ using a syringe pump, ionized by ESI, and analyzed using a Q-Exactive Orbitrap, quadrupole time-of-flight (QTOF), and linear ion trap (LTQ) MS. For the online derivatization, the capillary tip pulled to $\sim 25 \mu \mathrm{m}$ OD is illuminated with a green laser pointer $(532 \mathrm{~nm})$ positioned $15 \mathrm{~cm}$ away from the tip and focused using a convex lens. This configuration, which can be readily adapted to lipidomics experiments was used to test the feasibility of the online derivatization of $\mathrm{C}=\mathrm{C}$ bonds in fatty acyl chains using ${ }^{1} \mathrm{O}_{2}$ reaction.

In another experiment shown in Figures $1 \mathrm{c}$ and $\mathbf{S 1}$, the ${ }^{1} \mathrm{O}_{2}$ reaction is coupled to a highresolution nano-DESI probe to perform an on-line derivatization and $\mathrm{C}=\mathrm{C}$ profiling of analytes in complex lipid mixtures extracted from tissue sections. In this setup, the solvent containing RB is propelled through the nano-DESI probe comprised of the primary and spray capillary. Analytes are continuously extracted from a specific location on the sample into a dynamic liquid bridge formed between the two capillaries and surface as shown in the inset. The spray capillary transfers the extracted lipids and metabolites to a mass spectrometer inlet. The laser light is focused onto the tip of the spray capillary positioned at the MS inlet. A shear force probe is placed next to the nano-DESI probe to maintain a constant distance between the sample and the probe. ${ }^{[33-35]}$

The reaction between ${ }^{1} \mathrm{O}_{2}$ and unsaturated lipids (Figure 1a) generates an allylic hydroperoxide at either end of the double bond accompanied by a shift in the double bond position to the adjacent carbon. ${ }^{[31,32]}$ The reaction results in a mass shift of $32 \mathrm{Da}$ corresponding to the net addition of 2 oxygen atoms. We note that the presence of $\mathrm{Na}^{+}$in the RB salt used in these experiments results in formation of abundant sodium adducts of lipids in positive mode ESI. Multiple LOOH products are generated when ${ }^{1} \mathrm{O}_{2}$ reacts with multiply unsaturated lipids. Figure 2a shows mass spectra of a standard mixture containing LPE 17:1, PC 36:2 and TG 48:3 obtained upon irradiation. Although the dominant product corresponds to the addition of one $\mathrm{O}_{2}$, the distribution of the $\mathrm{LOOH}$ products observed in the spectrum confirms that the reaction occurs at each double bond. No other side products such as aldehydes, alcohols or ketones were observed in these experiments. CID of LOOHs generates unique neutral losses $(\mathrm{NL})$ indicative of the position of the hydroperoxide group and hence $\mathrm{C}=\mathrm{C}$ bond in the unreacted lipid. This is illustrated using two isomeric $\mathrm{LOOH}$ species generated from two positional isomers: PC 18:1(9Z)_18:1(9Z) and PC 18:1(6Z)_18:1(6Z) shown in Figure 2b. In this experiment, $1 \mu \mathrm{M}$ solutions of each standard containing $50 \mu \mathrm{M}$ RB were analyzed individually using ESI coupled with laser excitation. For both isomers, CID spectra shown in Figure 2c-d contain fragments at $m / z 781.4$ and 657.5 corresponding to head group losses from $\mathrm{m} / \mathrm{z}$ 840.5. However, fragmentation at the hydroperoxide generates different fragments for the two isomers. For the $9 \mathrm{Z}$ isomer, a fragment ion corresponding to the loss of $\mathrm{C}_{8} \mathrm{H}_{18} \mathrm{O}$ due to the 
cleavage at $\mathrm{C} 10$ of precursor $\mathrm{A}$ is observed at $\mathrm{m} / \mathrm{z}$ 710.4. This fragment undergoes subsequent NL of 59 and 183 generating peaks at $\mathrm{m} / \mathrm{z} 651.3$ and 527.3, respectively. The corresponding loss of $\mathrm{C}_{11} \mathrm{H}_{24} \mathrm{O}$ due to the cleavage at $\mathrm{C} 7$ is observed for the $6 \mathrm{Z}$ isomer generating a fragment at $m / z 668.4$ along with the head group losses at $m / z 609.3$ and $m / z$ 485.3. However, fragment ions corresponding to precursor B (Figure 1a) are not observed in the Orbitrap-CID spectra. These ions are observed as minor peaks in the LTQCID spectrum (Figure 2b). This effect is more pronounced for polyunsaturated LOOHs (Figures S2-S3), for which the diagnostic fragments of product $\mathrm{B}$ are observed in LTQ-CID but not in OrbitrapCID spectra. These results could be attributed to differences in the energetics and kinetics of fragmentation of the two $\mathrm{LOOH}$ products, which affect the branching ratio of the two expected product ions observed on different timescales and levels of internal excitation used by MS instruments employed in this study. Importantly, a fragment corresponding to product $\mathrm{A}$ is observed on all the instruments and can be used to identify the $\mathrm{C}=\mathrm{C}$ location of multiple lipid classes as shown in Figures S2-S6. For all glycerophospholipids, head group losses from both the precursor ion and the diagnostic fragments are observed in CID spectra. In contrast, TGs produce much cleaner CID spectra devoid of head group losses.

Optimization of the reaction conditions was conducted using a lipid extract from mouse gastrocnemius muscle tissue. The extraction procedure is described in the SI. Figure S7 shows mass spectra obtained at different concentrations of $\mathrm{RB}(5,50,100$, and $200 \mu \mathrm{M})$ in the solvent. We found that the $50 \mu \mathrm{M} \mathrm{RB}$ solution provided good yields of $\mathrm{LOOH}$ products without producing dominant RB-related peaks that suppress the ionization of lipids at higher RB concentrations.
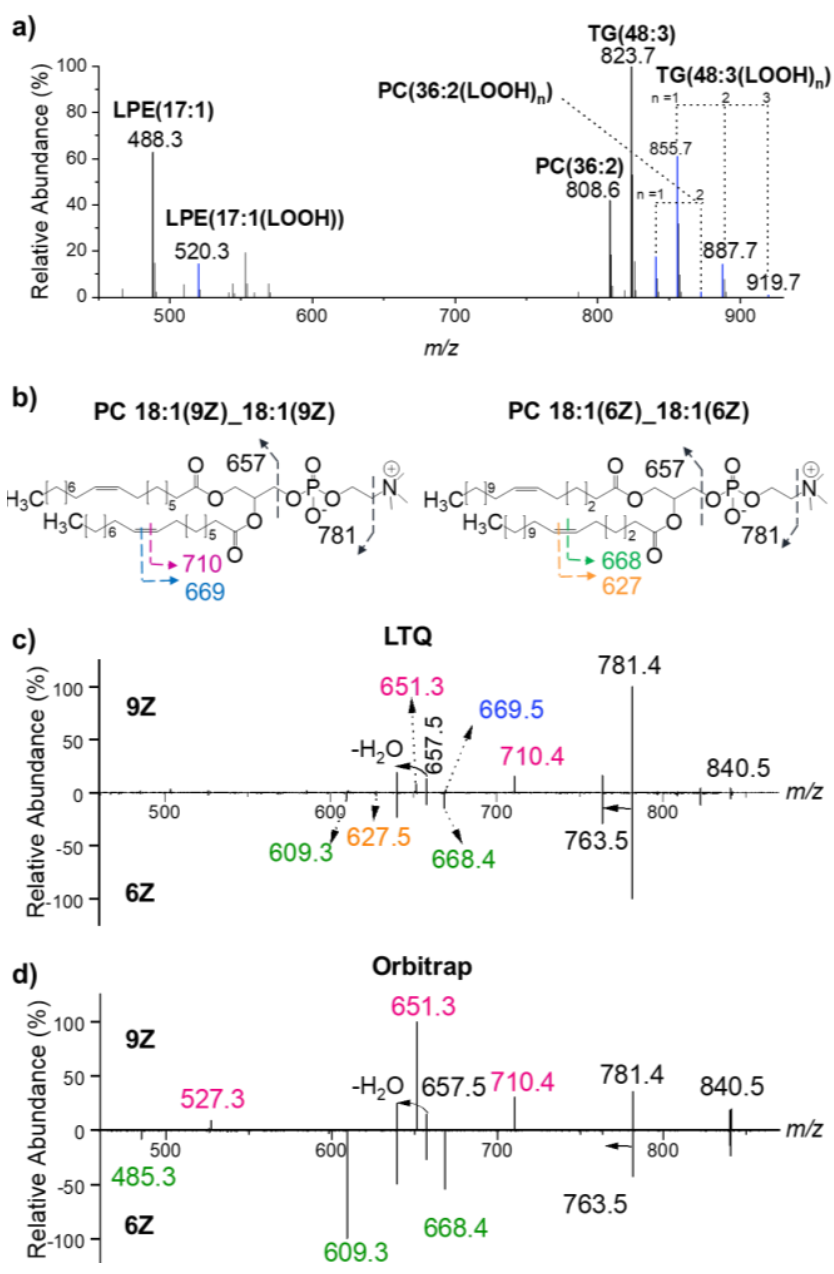

Figure 2. Elucidation of $\mathrm{C}=\mathrm{C}$ location in positional isomeric standards. a) A positive mode mass spectrum of LPE 17:1, PC 36:2 and TG 48:3 standards and their corresponding hydroperoxides, $(\mathrm{LOOH})_{n}$, generated by the reaction with ${ }^{1} \mathrm{O}_{2}$. The $(\mathrm{LOOH})_{n}$ species where $\mathbf{n}$ indicates the number of $\mathrm{O}_{2}$ additions are highlighted in blue. All the ions correspond to $[\mathrm{M}+\mathrm{Na}]^{+}$species. b) Structures of PC 18:1(9Z)_18:1(9Z) (left side) and PC 18:1(6Z)_18:1(6Z) (right side) showing the cleavage sites. CID spectra of the hydroperoxide products at $m / z 840.5$ of the isomeric standards acquired using c) LTQ and d) Orbitrap. 
Figure 3a shows part of a mass spectrum obtained under the optimized conditions, in which the $\mathrm{LOOH}$ products highlighted in blue exhibit a 32 Da mass shift from their precursor lipids. Ion chronograms of the endogenous PC 38:6 at $\mathrm{m} / \mathrm{z} 828.6$ (left) and PC 36:4 at $\mathrm{m} / \mathrm{z} 804.6$ (right) shown as black traces along with the corresponding $\mathrm{LOOH}$ products shown as blue traces are depicted in Figure 3b. The signals of PC 38:6(LOOH) at $\mathrm{m} / \mathrm{z} 860.5$ and $\mathrm{PC} 36: 4(\mathrm{LOOH})$ at 836.5 appear immediately after the light is turned on, reach a plateau within $\sim 3$ s showing a 30$40 \% \mathrm{LOOH}$ yield, and remain stable over time demonstrating the feasibility of this approach to perform online chemical derivatization of $\mathrm{C}=\mathrm{C}$ bonds. Using the solution flow rate and the estimated volume irradiated by the laser, we estimate the reaction time of $\sim 100 \mathrm{~ms}$ in these
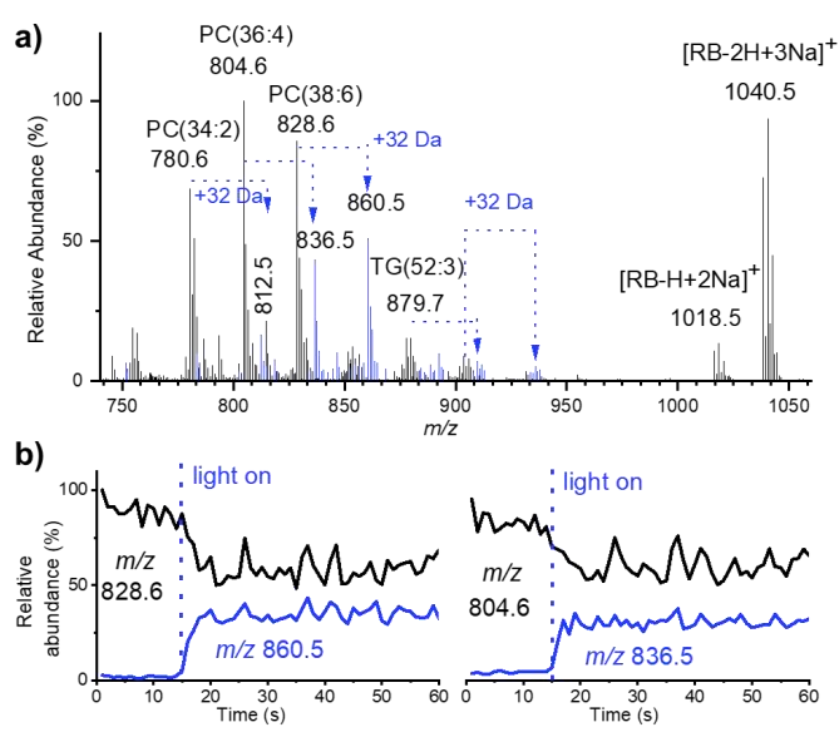

Figure 3. a) ESI-MS of a mouse gastrocnemius muscle tissue extract obtained using the SO reaction; the $\mathrm{LOOH}$ products are shown in blue. b) lon chronograms of the endogenous species at $\mathrm{m} / z 828.55$ (left panel) and $\mathrm{m} / z 804.55$ (right panel) and their corresponding reaction products at $\mathrm{m} / \mathrm{z} 860.54$ and $\mathrm{m} / \mathrm{z}$ 836.54, respectively, obtained with and without laser excitation. experiments. This rapid derivatization makes the ${ }^{1} \mathrm{O}_{2}$ reaction compatible with nano-DESI MSI experiments.

In order to demonstrate the feasibility of this approach for the spatially-resolved profiling or imaging of isomeric lipids directly from biological samples, we coupled the ${ }^{1} \mathrm{O}_{2}$ reaction with a high-resolution nano-DESI probe. The system was used for the spatial profiling of lipids in mouse uterine tissue sections. In this experiment, the sample is continuously moved under the probe at 20 $\mu \mathrm{m} / \mathrm{s}$ and mass spectra are acquired at $7 \mathrm{~Hz}$ acquisition rate. This experiment closely approximates imaging experiments performed using nano-DESI. The extraction solvent contains $50 \mu \mathrm{M}$ RB and $200 \mathrm{nM}$ of the LPE 17:1 standard. Before the nano-DESI probe is landed on the tissue, the standard is used to optimize the position and focusing of the laser by examining the yield of the hydroperoxide product, LPE 17:1(LOOH). Ion chronograms of LPE 17:1 and LPE17:1(LOOH) obtained with the nano-DESI probe operated in the air are shown in Figure S8 and demonstrate rapid $\mathrm{LOOH}$ formation upon laser excitation.

Figure 4 shows line scan profiles collected for the LPE 17:1 standard and an endogenous PC 34:1 extracted from the tissue along with the corresponding LPE 17:1(LOOH) and PC $34: 1(\mathrm{LOOH})$ products. When the nano-DESI probe is on a glass slide, the signal of the standard is high and other three molecules are not observed in the spectrum (Figure 4a). After the light is turned on, the ion signal of the LPE 17:1(LOOH) appears promptly (Figure 4b) while the signal of the unreacted LPE 17:1 drops. When the nano-DESI probe is on the tissue, the signals of the endogenous PC 34:1 and its product, PC 34:1(LOOH) are observed in the spectrum (Figure 4c- 
d). The correspondence between ion chronograms observed for these two species indicates that PC 34:1(LOOH) is generated with substantial yield on a timescale compatible with a typical nano-DESI MSI experiment. At $1 \mathrm{~min}$, the light is turned off, which causes the signal of the reaction products LPE 17:1(LOOH) and PC $34: 1(\mathrm{LOOH})$ to immediately disappear. Meanwhile, the signals of the corresponding precursor lipids are still detected. Finally, the signal of the endogenous PC 34:1 disappears when the nano-DESI probe gets off the tissue. At the same time, the signal of LPE 17:1 increases and reaches its initial value.

In situ CID profiling of lipids using a targeted list of $\mathrm{LOOH}$ products was performed while the nano-DESI probe was scanning the tissue. Table S1 lists fourteen unsaturated lipid species in mouse uterine sections, for which $\mathrm{C}=\mathrm{C}$ locations were successfully identified along with their

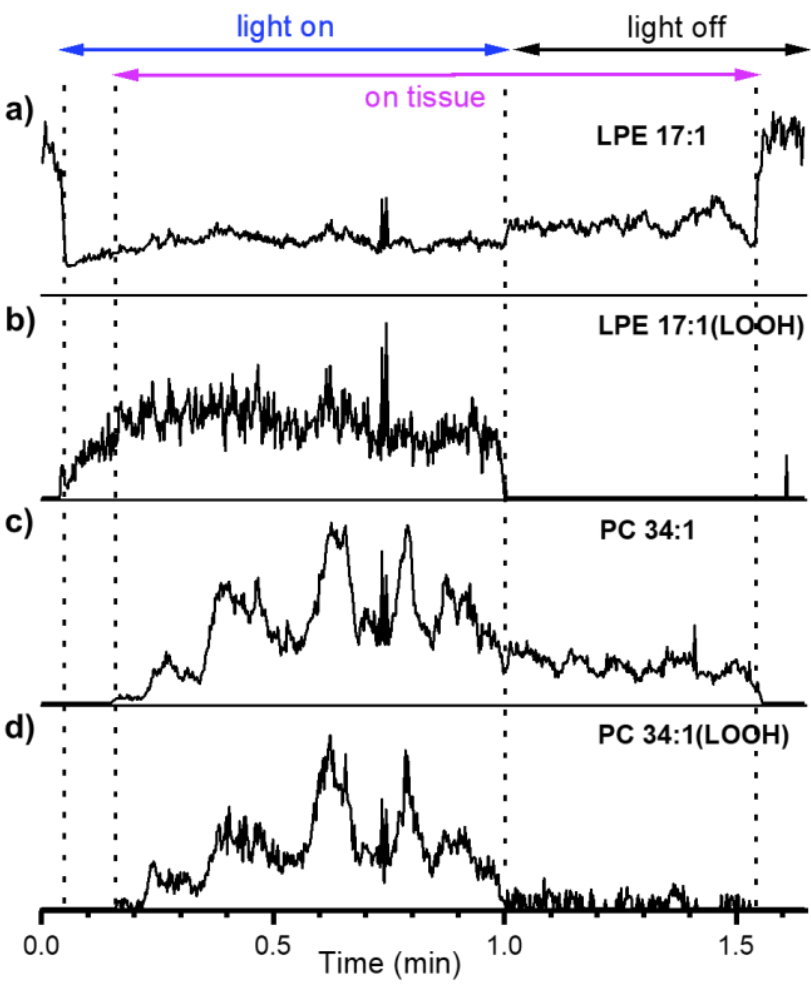

Figure 4. Line scan profiles collected with the nano-DESI probe over uterine tissue sections of a) std LPE 17:1 and b) endogenous PC 34:1 and the hydroperoxides b) LPE 17:1(LOOH) and d) PC 34:1(LOOH). diagnostic fragments. We found that lipids containing FA 18:1 showed the presence of the isomeric $9(Z)$ and 11(Z) pair. These isomeric species were identified based on CID spectra shown in Figure S9. This isomeric pair has been previously observed in different tissues, which validates our findings. ${ }^{[4,6]}$ It should be noted that due to the complexity of the lipidome, some acyl chain combinations may yield ambiguous identifications based on $\mathrm{MS}^{2}$ results alone. For example, the same neutral losses are expected for FA 16:1(7Z) and FA 18:1(9Z) or for FA 16:1(9Z) and FA 18:1(11Z). Therefore, lipids containing both FA 16:1 and FA 18:1 in the same molecule will require $\mathrm{MS}^{3}$ to unambiguously distinguish the $\mathrm{C}=\mathrm{C}$ bond locations. Nevertheless, a majority of positional isomers of lipid species can be readily identified based on $\mathrm{MS}^{2}$ experiments.

In summary, we have developed an online chemical derivatization method based on the ${ }^{1} \mathrm{O}_{2}$ reaction that allows identifying $\mathrm{C}=\mathrm{C}$ positions in different lipid classes. Rapid reaction rates enable on-the-fly photooxidation of lipids into their corresponding $\mathrm{LOOH}$ products which are detected promptly after laser excitation is turned on. The observed product yields and flow rates are consistent with a reaction occurring on a timescale of $\sim 100 \mathrm{~ms}$. CID of LOOH species produces unique neutral losses corresponding to the position of the hydroperoxide groups, which enables identification of the $\mathrm{C}=\mathrm{C}$ bond positions. This approach relies on an inexpensive light source and photosensitizer added to the solvent or analyte mixture and can be readily implemented on any mass spectrometer. As a result, it is compatible with both ESI-based lipidomics workflows and 
liquid extraction-based ambient ionization techniques and may be used for the spatial profiling of $\mathrm{C}=\mathrm{C}$ bonds in lipids extracted directly from tissue sections under ambient conditions. Coupling of the ${ }^{1} \mathrm{O}_{2}$ reaction with high-resolution nano-DESI imaging experiments is straightforward and will be explored in future studies. Collectively, our results indicate the power of the ${ }^{1} \mathrm{O}_{2}$ reaction for the rapid analysis of the $\mathrm{C}=\mathrm{C}$ bond positions in isomeric lipids, which will provide insights into their role in biological processes.

\section{Acknowledgments}

This research is supported by the grant from the National Science Foundation (NSF-1808136, JL) and National Institute of Health (HD068524 \& HD103475, SKD) and (R01AR071649, SK).

\section{References}

[1] T. Harayama, H. Riezman, Nat. Rev. Mol. Cell Biol. 2018, 19, 281-296.

[2] E. Fahy, D. Cotter, M. Sud, S. Subramaniam, Biochim. Biophys. Acta - Mol. Cell Biol. Lipids 2011, 1811, 637-647.

[3] X. Han, R. W. Gross, Mass Spectrom. Rev. 2005, 24, 367-412.

[4] W. Cao, S. Cheng, J. Yang, J. Feng, W. Zhang, Z. Li, Q. Chen, Y. Xia, Z. Ouyang, X. Ma, Nat. Commun. 2020, 11.

[5] D. R. Klein, C. L. Feider, K. Y. Garza, J. Q. Lin, L. S. Eberlin, J. S. Brodbelt, Anal. Chem. 2018, 90, 10100-10104.

[6] W. Zhang, D. Zhang, Q. Chen, J. Wu, Z. Ouyang, Y. Xia, Nat. Commun. 2019, 10.

[7] T. Porta Siegel, K. Ekroos, S. R. Ellis, Angew. Chemie - Int. Ed. 2019, 58, 6492-6501.

[8] S. H. J. Brown, T. W. Mitchell, S. J. Blanksby, Biochim. Biophys. Acta - Mol. Cell Biol. Lipids 2011, 1811, 807-817.

[9] D. L. Marshall, A. Criscuolo, R. S. E. Young, B. L. J. Poad, M. Zeller, G. E. Reid, T. W. Mitchell, S. J. Blanksby, J. Am. Soc. Mass Spectrom. 2019, 30, 1621-1630.

[10] C. E. Randolph, D. J. Foreman, S. K. Betancourt, S. J. Blanksby, S. A. McLuckey, Anal. Chem. 2018, 90, 12861-12869.

[11] J. L. Campbell, T. Baba, Anal. Chem. 2015, 87, 5837-5845.

[12] M. E. N. Born, B. M. Prentice, Int. J. Mass Spectrom. 2020, 452, 116338.

[13] D. R. Klein, J. S. Brodbelt, Anal. Chem. 2017, 89, 1516-1522.

[14] J. P. O'Brien, B. D. Needham, J. C. Henderson, E. M. Nowicki, M. Stephen Trent, J. S. Brodbelt, Anal. Chem. 2014, 86, 2138-2145. 
[15] X. Ma, Y. Xia, Angew. Chemie - Int. Ed. 2014, 53, 2592-2596.

[16] F. Tang, C. Guo, X. Ma, J. Zhang, Y. Su, R. Tian, R. Shi, Y. Xia, X. Wang, Z. Ouyang, Anal. Chem. 2018, 90, 5612-5619.

[17] K. A. Harrison, R. C. Murphy, Anal. Chem. 1996, 68, 3224-3230.

[18] Y. Feng, B. Chen, Q. Yu, L. Li, Anal. Chem. 2019, 91, 1791-1795.

[19] S. Tang, H. Cheng, X. Yan, Angew. Chemie - Int. Ed. 2020, 59, 209-214.

[20] S. Adhikari, W. Zhang, X. Xie, Q. Chen, Y. Xia, Anal. Chem. 2018, 90, 5239-5246.

[21] A. Bednařík, S. Bölsker, J. Soltwisch, K. Dreisewerd, Angew. Chemie - Int. Ed. 2018, 57, 12092-12096.

[22] M. R. L. Paine, B. L. J. Poad, G. B. Eijkel, D. L. Marshall, S. J. Blanksby, R. M. A. Heeren, S. R. Ellis, Angew. Chemie - Int. Ed. 2018, 57, 10530-10534.

[23] F. Wäldchen, B. Spengler, S. Heiles, J. Am. Chem. Soc. 2019, 141, 11816-11820.

[24] T. H. Kuo, H. H. Chung, H. Y. Chang, C. W. Lin, M. Y. Wang, T. L. Shen, C. C. Hsu, Anal. Chem. 2019, 91, 11905-11915.

[25] P. J. Roach, J. Laskin, A. Laskin, Analyst 2010, 135, 2233-2236.

[26] D. Unsihuay, J. Qiu, S. Swaroop, K. O. Nagornov, A. N. Kozhinov, Y. O. Tsybin, S. Kuang, J. Laskin, Int. J. Mass Spectrom. 2020, 448, 116269.

[27] M. Girod, E. Moyano, D. I. Campbell, R. G. Cooks, Chem. Sci. 2011, 2, 501-510.

[28] A. W. Girotti, J. Photochem. Photobiol. B Biol. 2001, 63, 103-113.

[29] I. O. L. Bacellar, M. S. Baptista, ACS Omega 2019, 4, 21636-21646.

[30] S. Kato, K. Nakagawa, Y. Suzuki, A. Asai, M. Nagao, K. Nagashima, S. Oikawa, T. Miyazawa, Anal. Biochem. 2015, 471, 51-60.

[31] J. Ito, S. Mizuochi, K. Nakagawa, S. Kato, T. Miyazawa, Anal. Chem. 2015, 87, 49804987.

[32] S. Kato, N. Shimizu, Y. Hanzawa, Y. Otoki, J. Ito, F. Kimura, S. Takekoshi, M. Sakaino, T. Sano, T. Eitsuka, T. Miyazawa, K. Nakagawa, Sci. Food 2018, 2.

[33] S. N. Nguyen, A. V. Liyu, R. K. Chu, C. R. Anderton, J. Laskin, Anal. Chem. 2017, 89, 1131-1137.

[34] S. N. Nguyen, R. L. Sontag, J. P. Carson, R. A. Corley, C. Ansong, J. Laskin, J. Am. Soc. Mass Spectrom. 2018, 29, 316-322.

[35] R. Yin, K. E. Burnum-Johnson, X. Sun, S. K. Dey, J. Laskin, Nat. Protoc. 2019, 14, 3445-3470. 